\title{
Carnets
}

Revue électronique d'études françaises de l'APEF

Deuxième série - $18 \mid 2020$

Chiens et écritures (littéraires, filmiques, photographiques)

\section{Pour un chien jaune}

Questions de figuration humaine au cinéma

Jean-Michel Durafour

\section{(2) OpenEdition}

Journals

Édition électronique

URL : http://journals.openedition.org/carnets/10491

DOI : 10.4000/carnets.10491

ISSN : 1646-7698

Éditeur

APEF

Référence électronique

Jean-Michel Durafour, "Pour un chien jaune », Carnets [En ligne], Deuxième série - 18| 2020, mis en ligne le 31 janvier 2020, consulté le 02 juin 2020. URL : http://journals.openedition.org/carnets/10491 ; DOI : https://doi.org/10.4000/carnets. 10491

Ce document a été généré automatiquement le 2 juin 2020.

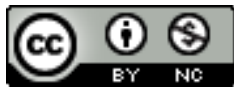

Carnets est mis à disposition selon les termes de la licence Creative Commons - Atribution - Pas d'utilisation commerciale 4.0 International. 


\title{
Pour un chien jaune
}

\author{
Questions de figuration humaine au cinéma
}

\author{
Jean-Michel Durafour
}

Débarqué aux Antilles, très précisément dans la baie de l'actuel Cuba, Christophe Colomb est invité par les Arawak à se méfier du peuple caribe, spécialement belliqueux. Dans la langue des Petites Antilles, cariba signifie "hardi». Les Européens des pays latins le déformèrent par la suite en caniba.

Colomb décrit ainsi les Caribes, qu'il n'a pas vus de ses yeux : « un seul œil et une face de chien » (Colomb, 1984 : 117). La superposition de l'imaginaire occidental de l'époque associé aux chiens errants se nourrissant de cadavres humains (maladies, guerres) sur une source amérindienne, tous deux lourdement chargés d'anthropophagie, a engendré un mot complexe - canibalismo - où manger de la chair humaine revient à avoir une tête de chien (canis). Une gravure de 1525 de Lorenz Fries, tirée de son ouvrage Carta marina, montre explicitement des hommes à têtes de chien dépecer non pas des hommes, mais d'autres corps humains.

Dans l'exemple du cannibalisme, la référence au chien sert deux objectifs, dont je crois qu'ils sont également immanents au cinéma photographique, un médium avaleur de chair fraîche et dévoreur de corps. Le film - on comprendra donc que je ne parlerai ici que du cinéma en prise de vues réelle - est une image anthropophage. On verra progressivement qu'il est également en mesure d'accomplir les exploits visuels d'une image canine.

4 Ce double programme est le suivant : parler de l'homme, c'est-à-dire parler de l'homme comme problème. Figurer conjointement l'homme et le chien, penser figurativement l'un par l'autre, c'est attester d'une énigme dans la présentation de l'homme. Selon les cas: cet homme, ces hommes, tout homme, etc. Entre autres milliers d'exemples : des hommes qui en mangent d'autres, mais aussi un mendiant qui cache un roi (le retour d'Ulysse à Ithaque) ou, pour en venir à de premiers exemples cinématographiques, un homme qui en réalité est un spectre (L'Aventure de $M^{m e}$ Muir [1947] de Mankiewicz), un homme qui vient d'être mordu par un loup-garou (Le Loup-garou [The Wolf Man, 1940] de George Waggner), et ainsi de suite. Les énigmes sont de toutes sortes : leur trait commun est d'être des énigmes. Le chien par son comportement - fuite, grondement, morsure - 
signale qu'une figure humaine interroge, en deçà des apparences aspectuelles anthropomorphes, sinon la nature de l'homme (il n'y en a sans doute aucune), mais les conditions de l'homme. Dans tous les sens qu'on voudra bien donner à cette expression : ses circonstances, ses préalables, ses injonctions.

5 À qui la Sphinge pose-t-elle d'ailleurs la question de l'homme, si ce n'est à đEdipe, c'està-dire à l'homme qui a le "pied [podos] enflé [oidi]» et qui, parce qu'il a le pied tel aurait la capacité d'entrevoir, de voir en devinant (oida) ? Et pourquoi a-t-il le pied enflé, notre đEdipe, à qui le berger chargé de le tuer enfant avait percé le talon pour l'accrocher à un arbre par une cordelette, si ce n'est parce que c'est d'abord aux chiens que l'on faisait alors subir ce traitement du tarse pour les empêcher de rompre leurs chaînes ? Pourquoi a-t-il le pied enflé, si ce n'est parce que celui qui peut trouver la réponse à la question de l'homme, celui qui est suffisamment malin, retors, le premier à ne pas avoir un mal de chien à répondre, celui qui possède l'excellence des qualités de l'esprit humain tout en représentant la condition humaine la plus tragique dans sa ridicule démesure, ne peut être qu'un chien?

6 Je ne m'intéresserai pas ici à la figuration des chiens pour eux-mêmes - de toutes sortes au cinéma: de compagnie, errants, de cirque, de battues policières, de laboratoire, d'aveugle, des Baskerville, de races, dogues, roquets, clébards, etc. - mais, en prolongeant ce propos liminaire, dans la " double capture ${ }^{1}$ " visuelle entre l'homme et le chien en regard de la question de l'homme. Mon hypothèse principale est la suivante : si le chien est une invention de l'homme, par des siècles de croisements à partir du loup depuis la préhistoire, s'il est un mélange de nature et de technologie (Ed Russell), le cinéma aura contribué à faire voir comme jamais auparavant que l'homme peut être une invention figurative et plastique à partir du chien. Car, à bien des égards, la domestication n'a rien d'une maîtrise unilatérale : le chien a largement aussi domestiqué l'homme, concourant à faire de lui une espèce " civilisée ", en même temps qu'il a su s'adapter à l'homme de lui-même (sans intentionnalité particulière) en adoucissant le tempérament du loup et en affaiblissant son instinct de fuite. Comme le note Keith Thomas (Dans le jardin de la nature), la domestication a peu à peu transformé les animaux en «membres subsidiaires de la communauté humaine " (Thomas, 1985 : 128) - on prénomme les chiens, on les enterre avec cérémonie, on leur confie la garde de la maison ou du troupeau, on les nourrit parfois mieux que des hommes, on leur confère un statut social (bâtards, limiers, etc.) -, en même temps que pendant très longtemps il y a eu des chiens partout (dans les fermes, à la cour, chez les aristocrates) et que les hommes ont été façonnés par leurs contacts avec les chiens: "Certains hommes ne peuvent s'écarter d'un demi-mille de chez eux s'ils n'ont pas un chien sur leurs talons » (Bunyan, 1842 : 15). L'anthropologie atteste partout de la symbiose "chorégraphique » (Thompson, $2005:$ 42) de l'homme et du chien.

7 Homme et chien ne forment qu'un seul être relationnel par spéciations mutualistes et multiformes. La philosophe et biologiste Donna J. Haraway embrasse sa chienne, $\mathrm{M}^{\text {lle }}$ Cayenne Pepper, à pleine bouche. Elles ne sont qu'un corps hybride et impur, dans lequel la nature et la culture sont intrinsèquement enchevêtrées. J'en retiens que le chien n'est pas qu'un animal de compagnie, c'est une "espèce compagne ", pour traduire correctement en français le titre de l'ouvrage d'Haraway de 2003 Companion Species Manifesto (et non " espèces de compagnie »). Les hommes et les chiens ont noué des rapports "plein de gâchis, de cruauté, d'indifférence, d'ignorance et d'abandon, mais aussi de joie, d'invention de travail, d'intelligence et de jeu » (Haraway, $2010: 19$ ), 
qui dessinent « un bestiaire de capacités d'agir, de formes de mise en relation et de faisceaux de temps défiant jusqu'à l'imagination du plus baroque des cosmologues » (idem: 14). Je m'intéresserai ici à la dimension figurative de cette "natureculture " (idem: 10) anthropozoologique, en m'attachant à la manière dont elle peut se rencontrer au cinéma sous les espèces d'un cinéma canin, au sens proche de l'« écriture canine " (idem: 11) - mais non réduite à la théorie féministe - par laquelle l'être humain trace également sa figure écrite par le prisme du chien (le journal intime "Chronique d'une fille de journaliste sportif »)2.

Chien et homme sont des espèces dont la coévolution s'est prolongée, renouvelée et amplifiée dans une co-iconicisation ${ }^{3}$. Le cinéma, après la peinture et la sculpture, la gravure et la photographie, l'a - tout autrement - pris aussi à sa charge. Et peut-être mieux que toute autre image: art par excellence ovidien (nous ne cessons de commercer avec les formes des animaux) et darwinien (tout n'est que variations de variations), il n'aura mis en scène que des changements de formes dans d'autres, en autres, par d'autres - et sans doute à mieux dire : qu'il n'y a partout qu'une seule forme qui change, comme nous ne voyons à l'écran qu'une seule image qui bouge - dans la fête dispendieuse des métamorphoses, l'entropie préparée des mutations et le mystère insituable du visible. C'est ce que je propose de nommer pour l'occasion, en empruntant la formule au titre d'un célèbre roman de Georges Simenon, le "chien jaune" (Simenon, 1931) : ce chien dont la présence quasi surnaturelle annonce à chaque fois le crime humain. Comme je serai amené à le montrer, au centre de mon propos se tient le postulat que l'homme est un chien décliné. Là encore dans toutes les acceptions parfois contradictoires que ce terme implique pour qui pense en français : enrichir en variant les formes (décliner un substantif latin ou un produit commercial) ou bien diminuer (être sur le déclin), refuser; s'approcher dans la perception d'un horizon hors d'atteinte.

9 Le cinéma a un lien privilégié avec l'animal. Et ce, pour des raisons qui sont très bien analysées par Étienne Souriau dans son article de 1956 "L'univers filmique et l'art animalier ». L'auteur y rappelle que le développement du cinéma a précisément eu lieu à un moment où, par des causes plurielles (environnementales, industrielles), notre lien culturel à l'animal s'est distendu: le cinéma coïncide avec «la cessation brusque et presque totale du cheval comme force motrice, comme procédé pratique de locomotion, comme moyen de combat » (Souriau, 1956:53), en même temps que ce même cheval, non sans ironie, a joué un rôle décisif dans la décomposition du mouvement en images photographiques animées (chez Muybridge puis Marey), c'est-àdire dans la généalogie qui conduira vite au cinéma. Souriau indique ensuite les trois modalités figuratives sous lesquelles, selon lui, l'animal s'impose au cinéma " en son autonomie » (Souriau, 1956: 58) : cinétiques, morphologiques, expressives. Dans la lecture que je propose ici, il existe trois manières principales - je n'en exclus pas d'autres - de figurer le «chien jaune" dans une coévolution à l'homme qui fait du chien le portrait de l'homme (et de l'homme du chien : mais je laisse ici ce problème de côté), comme Deleuze parle de la toile d'araignée en "portrait très subtil de la mouche» (Deleuze et Guattari, 1991: 175). Je les nomme pour l'occasion: anthropologique, anthropomorphologique, anthropocéphale. Ils recoupent partiellement la trilogie sourialienne: aux chiens anthropologiques les qualités de l'expression, aux anthropomorphiques celles de la morphologie; aux anthropocéphales celles des mouvements (isolés des corps mobiles). De ces trois catégories, la troisième seule peut donner lieu à des modes de représentation exclusivement cinématographiques. Les 
deux premières se rencontrent également dans les autres arts, notamment la peinture ou la littérature, mais bien entendu par d'autres champs d'énergie figurative.

\section{Le chien anthropologique}

\section{humains (franchise, loyauté, etc.) des propriétés ou des états de l'homme. C'est peut-être, dans} tout le règne artistique, le cas le plus répandu.

Il s'agit, par le chien, de symboliser un élément de la spécificité de l'homme par rapport aux animaux, c'est-à-dire comme être moral. On lui doit - entres autres - l'image d'Épinal du chien nigaud, de la bêtise du chien (la bêtise est humaine, la bête n'est pas bête), qui fait tant de mal à l'animal : l'obéissance aveugle même pour un mauvais maître, le confort avant l'indépendance, le plaisir dans la soumission, la passion de l'intégration ${ }^{4}$. « La joie du chien, sa jouissance, est en ce sens non seulement une énigme, mais une sorte de scandale. Elle choque l'idée qu'on peut se faire d'une dignité qui devrait se fonder sur le “mérite": le labeur de l'intelligence, l'effort de la liberté » (Alizart, $2017: 21-22^{5}$ ). Sa version dépravée sont les chiens infernaux, ici ou là, comme le cénobite canin de Hellraiser 4 (Hellraiser: Bloodline, 1996) de Kevin Yagher, ou les chiens possédés, contaminés, «estrangés » qui se retournent contre les humains : Suspiria (1977) de Dario Argento, L'Au-delà (1980) de Lucio Fulci, The Thing (1982) de John Carpenter, Cujo (1983) de Lewis Teague (d'après Stephen King). Le cinéma fantastique ou d'horreur en a fait des gorges chaudes.

12 Le chien anthropologique n'est pas figurativement aligné sur la forme de l'homme: ses postures sont canines, il ne parle pas, et ainsi de suite. En revanche, c'est une certaine anthropomorphisation discursive du chien (parler de dévouement à son propos, par exemple : lire son comportement à partir d'une grille de lecture humaine) qui rend possible a priori ce retour figuratif à l'homme par le chien. Je reviens plus loin sur la question (visuelle) de l'anthropomorphisme.

13 C'est ce que l'on peut trouver en peinture dans La Vision de saint Augustin (1502-1507) de Carpaccio. Assis à sa table de travail, l'évêque d'Hippone, qui vient d'accomplir son œuvre, lève la plume et regarde désormais vers l'irreprésentable. Très traditionnellement, le chien personnifie ici la vertu théologale de la foi. Van Eyck (Les Époux Arnolfini) ou le Titien (Portrait d'Éléonore Gonzague) l'ajoutent à leurs portraits pour signaler la fidélité du mari ou de la femme.

Et au cinéma?

Dans Umberto D. (1952) de Vittorio De Sica, un modeste fonctionnaire à la retraite (Carlo Battisti) essaie de joindre les deux bouts pour se loger avec son chien Flike dans une modeste pension. Petit à petit abandonné de tous et réduit à la mendicité, il se décide à confier son compagnon à quatre pattes, un bâtard de race indéterminée, lui aussi un moins que rien de l'espèce (les critères sont sociaux pour l'homme; biologiques pour l'animal). Mais le chien revient toujours à son maître et l'empêchera finalement de se suicider. Ou du moins, c'est ainsi que le film se termine : car, après qu'Umberto aura été traversé par l'idée se jeter sous un train avec Flike, que ni les gardiens ni la petite fille du jardin public ne parviennent à retenir près d'eux, le chien se fera méfiant, reculera à son approche, échaudé par la trahison impardonnable... qu'il finira par pardonner. Il faudra le jeu final avec la pomme de pin pour qu'homme et chien s'apprivoisent de 
nouveau par la «mimétique » (Bellour, 2009 : 546). La joie du chien et sa fidélité à toute épreuve témoignent en creuset de l'inhumanité des hommes (avidité des usuriers, pingrerie de la tenancière, hypocrisie des nonnes, indifférence des proches) et le chien - comme c'est souvent son rôle dans le monde animalier - sauve la vie de l'homme, ici par l'évidence des délices simples de la vie par une journée ensoleillée, au milieu des cris d'enfants. Le chien tient ici le rôle anthropologique du don de soi et de la bonté désintéressée.

Autre exemple. Dans L'Impossible Monsieur Bébé (Bringing Up Baby, 1938) de Howard Hawks, un savant est d'emblée promis à un mariage sans enthousiasme avec sa secrétaire présentée comme une jeune femme austère et de toute évidence frigide, à laquelle il n'est liée que par le travail. Cette union n'est que le prolongement par d'autres moyens d'une activité professionnelle tout entière dévolue à l'étude paléontologique, c'est-à-dire d'une vie passée parmi des existences fossiles (pas de lune de miel, pas d'enfant). Le début du film multiplie les allusions grivoises à l'absence de rapport sexuel entre les fiancés, comme lorsque David (Cary Grant) se plaint devant sa promise de n'avoir pas réussi la veille à entrer, comme il l'espérait, un os (a bone) dans la queue d'un squelette de dinosaure, à savoir dans le derrière, c'est-à-dire à avoir une érection (a boner). Quand Susan (Katharine Hepburn), une mondaine écervelée et pétulante, ravagera la routine de David, ils se rapprocheront l'un de l'autre à partir du moment où ils devront courir après un autre os de dinosaure important, dernière pièce d'un squelette de brontosaure, escamoté par un fox-terrier à nom d'homme (il s'agit d'une "clavicule intercostale», os purement imaginaire et symbolique, puisqu'il reviendrait pour son propriétaire à avoir la tête sous les pattes avant ${ }^{6}$ ). Ils vont tomber amoureux l'un de l'autre parce que, pour la première fois, David va rencontrer une compagne, une " compagne d'espèce ", qui l'aidera à trouver son érection (voir, un peu avant, la scène ébouriffante du restaurant où David, qui vient par inadvertance de déchirer le dos de la robe de Susan, colle à ses fesses pour en dissimuler le trou aux regards des dîneurs, en venant s'emboîter sur elle par derrière). Le chien est le médiateur érotique qui déplace leurs premières relations conflictuelles vers le jeu sexuel entre partenaires, actualise un art de la conversation et de l'amour, de la conversion du scepticisme en reconnaissance de l'autre ${ }^{7}$.

L'éventail anthropologique du chien est très vaste - c'est même, des trois, la catégorie du « chien jaune » la plus présente au cinéma: danger pour l'humain dans Dressé pour tuer (White Dog, 1982) de Samuel Fuller; avertisseur d'un danger pour l'humain dans Amityville: La Maison du diable (The Amityville Horror, 1979) de Stuart Rosenberg ; témoin de notre mort dans Le Procès de Jeanne d'Arc (1962) de Robert Bresson ; première victime du bonheur familial menacé dans Funny Games (1997) de Michael Haneke ou Les Nerfs à vif (Cape Fear, 1962) de Jack Lee Thompson; substitut d'enfant dans Cette sacrée vérité (The Awful Truth, 1937) de Leo McCarey; dernier compagnon de l'homme après l'apocalypse dans Mad Max 2: Le Défi (Mad Max 2: The Road Warrior, 1982) de George Miller ou Je suis une légende (I Am Legend, 2007) de Francis Lawrence; instrument d'une vengeance dans Les Yeux sans visage (1960) de Georges Franju; souffre-douleur de la brutalité des hommes dans Post Tenebras Lux (2012) de Carlos Reygadas, etc. 


\section{Le chien anthropomorphologique} humaine, des propriétés ou des états du corps de l'homme comme corps biologique et pathique en général. Il s'agit cette fois-ci, par le chien, de marquer la non-rupture de l'homme avec le reste du vivant, son absence de spécificité comme corps animal, c'est-à-dire non humain. L'on n'y prend plus l'homme par l'exception (l'âme, la morale), par l'animal qu'il n'est pas, mais par le dénominateur commun du corps zoologique. Ce régime décalque par le chien que le cinéma ne s'est jamais posé qu'une seule question: comment fabriquer l'homme pour qu'il ressemble le plus à l'homme, et ce, y compris, et peut-être surtout quand il n'est pas à l'image, quand l'homme se ressemble si peu à luimême?

Précision importante. - On prendra soin de ne pas confondre l'anthropomorphologisme avec l'anthropomorphisme, qui est absolument éloigné de mon propos. Celui-ci ne tient pas compte de l'animal, qu'il replie univoquement sur des procédures humaines : par

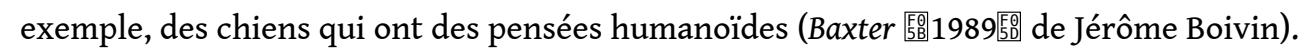
C'est l'anthropomorphisme qui nous conduit notamment à hiérarchiser entre les animaux qui paraissent avoir un visage, vivre en étroite association symbiotique avec l'homme ou exprimer des émotions que nous éprouvons d'abord parmi nous, et ceux à qui il manque l'homme (cafard, reptile, etc.), et avec lesquels nous faisons généralement preuve de nettement moins d'égards car ils ne nous regardent pas. L'anthropomorphisme - qui a aussi fait florès au cinéma, notamment dans le dessin animé américain (Dingo, Pooch the Pup, Droopy) ou dans le film animalier pour jeune public et son cortège de chiens stars (Rintintin, Lassie) - ne s'intéresse aux animaux qu'à proportion de ce que l'on y repère singer les hommes, de ce qui est encore de l'homme; l'anthropomorphologisme, quant à lui, figure les animaux comme des vecteurs en corps avec l'homme, c'est-à-dire dans un dépassement imaginaire de la distinction entre humains et non-humains (que l'anthropomorphisme ne peut que reconduire). L'anthropomorphisme est le masque de l'exceptionnalisme humain, qui n'est que le symétrique du réductionnisme biologique dans la mécompréhension de la complexion canine.

Les chiens anthropomorphologiques ne sont pas exclusifs du cinéma. La peinture de Lucian Freud, par exemple, en réserve de nombreux. Sunny Morning - Eight Legs (1997) ou Double Portrait (1986) instaurent figurativement les corps humains et canins en mutations d'une même matrice formelle absente. Dans Double Portrait, le couple formé par Bella (l'une des filles du peintre) et le whippet Joshua, allongés côte à côte, est moins la coexistence de deux formes distinctes rapprochées dans l'espace, par une pensée chosique et centripète de la peinture (analogie, ressemblance), qu'un réseau centrifuge de variations morphologiques, de conjugaisons successives rapportant femme et bête à un seul corps organique bourgeonnant, pyrétique. La pliure du coude de la femme prolonge la courbure du crâne du chien, ou inversement - il n'y a ici aucun ordre chronologique - non pas parce que l'une renverrait à l'autre, mais parce que l'une et l'autre, jusque dans les moindres détails (les veines bleutées des mains et des avant-bras répondent exactement aux tâches sur le pelage de l'animal), saillissent de la plissure irreprésentable du sommeil, des corps chiffonnés, des vigilances froncées, des états de conscience anesthésiés, dans laquelle l'homme ne se distingue plus de l'animal, sorte de somnambules permanents, et comme lui ne fait plus qu'un avec la nature 
universelle, «âme sentante dans son immédiateté » (Hegel), en un corps indivisible à peine perceptible entre des corps factuels devenus trop indigents pour ne pas exploser dans une apothéose de vecteurs. (Au cinéma, on en retrouverait un exemple très proche dans Une vie de chien [A Dog's Life, 1918] de Chaplin, lorsque le vagabond accommode son compagnon d'infortune en oreiller de circonstance).

21 J'en prendrai plus en détail un seul exemple filmique. Adresse inconnue (Suchwiin bulmyeong, 2001) de Kim Ki-duk met en scène trois locaux de Pyongtaek, un village (kijich'on) vivant de la présence américaine militaire depuis la guerre de Corée (maisons closes, bars, etc.). Le film montre d'abord plusieurs chiens battus et destinés à l'alimentation ${ }^{8}$. Certains plans sont particulièrement éprouvants. On peut commencer par appréhender le sort de ces chiens dans la perspective anthropologique de tout à l'heure : face à des êtres humains ayant abdiqué toute humanité (copulations bestiales, scarifications insupportables), les animaux torturés deviennent - au-delà de la question extra-cinématographique du traitement inhumain, c'est-à-dire indigne d'êtres humains, que l'on réserve aux animaux - le seul point de la représentation venant provoquer la compassion du spectateur, l'infinie capacité à être une victime, c'est-à-dire un être humain (il n'y a pas de victime dans la prédation animale: la victime suppose l'injustice, or rien n'est injuste dans les lois de la nature ${ }^{9}$ ), à retourner l'humanité en une qualité de l'animal ${ }^{10}$.

22 Mais il y a plus. Les êtres humains du film, pour leur part, tous déviants, ne seront en mesure d'éveiller notre sympathie qu'à mesure, non seulement de ce qu'ils aimeront les chiens, mais surtout de ce qu'ils seront filmés, non pas comme des chiens (la métaphore est toujours une extériorité), mais dans un composé morphologique avec le chien. Entre les personnages circulent un certain nombre de motifs - narratifs ou sensibles - associant les deux figures principales visuelles du film, le chien et l'œil humain, comme par exemple dans la relation entre le boucher canin, surnommé " đil de chien», et son beau-fils. Un chien joue un rôle important dans ce complexe : le chiot de l'adolescente borgne. Dans une scène - il faut bien l'avouer - troublante, Eun-ok (Park Min-jung) se donne du plaisir en frottant l'animal contre son sexe, après l'avoir toiletté et séché contre sa poitrine, tandis qu'un jeune apprenti-peintre amoureux d'elle l'observe à son insu depuis un œilleton pratiqué dans une cloison en papier. Dans deux autres passages d'Adresse inconnue, la jeune femme avoue « se sentir chienne » en regardant des chiens des rues forniquer et trouve que les magazines pornographiques font « ressembler les couples à des chiens». Dans la scène suscitée, la figuration ne choisit pas de faire mimer le comportement canin par l'espèce humaine (on imite quelque chose ou quelqu'un que l'on n'est pas) : elle connecte en une seule figure anthropocynologique, sans solution de continuité, les corps du chien et de la femme par les vases communicants de leurs biologies intimes (vagin, gueule). Chacun est le prolongement figuratif de l'autre en un composé animal « symbiogénétique » (Haraway, $2010: 9$ ). Voir dans cette scène un moment zoophile, c'est regarder par l'autre bout de la lorgnette, par le mauvais trou dans le mur ou le mauvais œil, c'est isoler, en les extériorisant l'une l'autre, chacune des formes d'un composé qui ne tient que par l'indifférenciation spéciée des corps de l'homme et de l'animal. Cette scène n'est pas plus zoophile qu'elle est anthropophile. C'est-à-dire ni l'un ni l'autre.

L'anthropozoologie morphologique invite à écarter l'homme qui vit dans nos yeux. À voir, à penser avec une tête d'animal. Une tête de chien. Ce qui, rappelons-nous la première propriété du devenir-animal deleuzien que nous recroisons autrement, ne 
veut absolument pas dire devenir un animal, se changer en chien, voir comme un chien voit, etc. - cela supposerait une transcendance, c'est-à-dire une extériorité, et que l'homme ne soit plus l'homme à partir d'un certain point - mais signifie, puisque c'est l'homme qui devient et n'en finit jamais de devenir, voir, penser au lieu du chien (Durafour, 2013 : 33-34), dans tous les sens avérés de l'expression en français : voir d'où voit le chien parce que l'on voit en lieu et place du chien, que l'on continue de voir en homme. «Le devenir-animal de l'homme s'avère réel sans que soit réel l'animal qu'il devient » (Deleuze et Guattari, $1980: 291^{11}$ ).

\section{Le chien anthropocéphale}

De la modalité figurative anthropocéphale qui va désormais venir, je dirai pour commencer que l'on va avoir affaire à une image qui «a du chien ». Qu'est-ce que cela veut dire, « avoir du chien »? D'abord, au sens littéral, c'est une image qui a du chien, dans laquelle il y a un ou des chiens. Mais c'était - bien entendu - déjà le cas des images précédentes. C'est parce que les images dont je parle ont des chiens, qu'il y a à voir $d u$ chien, non seulement dans l'image, mais surtout dans le comment de l'image, dans l'injonction de ses procédures figuratives. Une image qui a du chien, c'est donc autre chose, ou qui montre les rapports figuratifs hommes-chiens peut-être d'une manière très différente de ce que nous connaissons pour l'instant. C'est une image qui exerce un charme particulier, comme on peut dire familièrement d'une femme qu'elle « $a \mathrm{du}$ chien ", un je-ne-sais-quoi, c'est-à-dire un presque-rien (Jankélévitch) - elle a du rien qui fascine tout en étant un peu provocateur. Mais toutes les images n'ont-elles pas un charme plus ou moins affolant et aguicheur? Ce qui attire l'attention dans une image qui a du chien, c'est surtout que l'image y tient du chien. Il y a du chien dans cette image. Non seulement, comme on a vu précédemment, il peut y avoir du chien dans l'être humain, dans le composé zooanthropologique à l'image - «Un homme en image - en forme d'animal » (Eisenstein, $2013: 65$ ) -, mais il y a du chien dans l'image, présent à l'écran ou non.

Pour ce qui est de la question qui m'occupe présentement, celle de la cofiguration de l'homme et du chien (tout le contraire d'une configuration, d'un réglage), de l'un par l'autre, nous allons rencontrer ce que je propose de nommer le chien anthropocéphale.

Ce cas possède aussi sa variante anthropomorphique avec laquelle on prendra soin de ne pas le confondre : l'homme cynocéphale, mieux connu, d'Anubis et saint Christophe de Lycie (dans la tradition orthodoxe) au clip de « Da Funk » réalisé pour Daft Punk par Spike Jonze en 1997, en passant par les voyages de Marco Polo ou la mythologie chinoise. Ici, la tête de chien vient en complément d'une base humaine, et n'est qu'un masque: le composé se comporte comme un homme, il se tient debout, porte des vêtements d'homme, lit, écoute de la musique, etc. On peut également évoquer, dans le même sens, tout ce que l'on trouvera dans la littérature de science-fiction autour des chiens « évolués » qui parlent, raisonnent et ont remplacé les hommes dans l'avenir de l'évolution des espèces - chez Cordwainer Smith (Les Seigneurs de l'instrumentalité: les chiens sont devenus des "Hommes Non Autorisés» qui feront redécouvrir aux descendants de l'«Homme Véritable» la religion chrétienne), chez Clifford Simak (Demain les chiens : une série de contes racontés par des chiens et commentés par trois exégètes canins qui en discutent l'authenticité). 

contrario poser que l'homme est une tête d'homme sur un corps animal, en l'occurrence de chien, qui dessine à la fois une généalogie et un programme de figures, et dans le même temps s'interroger sur les inventions de l'image par lesquelles ces devenirs sont visuellement possibles, dans la mesure où la frontière entre humain et non humain ne peut passer que dans l'homme. L'homme va se déduire désormais figurativement du chien, en deçà de tout propos moral ou biologique, dans l'absentement qu'est toute figure. L'image anthropocéphale est une animalisation de l'homme et de l'image de l'homme.

(La philosophie nous l'aura déjà enseigné : si je me distingue des autres animaux par la pensée rationnelle qui n'est que l'attribut de l'homme, si je conçois, c'est parce que je suis un chien. L'autre sens du mot concevoir - enfanter - ne dit rien d'autre : en grec ancien, kuon [le chien] est très proche de kuô [être enceinte]. On y retrouve le principe même de la maïeutique socratique : l'homme sage est avant tout une sage-femme, qui fait accoucher les autres âmes sans accoucher lui-même ; c'est un chien [Platon, 1993 : $109^{12}$. Les Cyniques, qui ne doivent au départ leur surnom de « chiens » que d'un jeu de mots [ils se réunissaient au Cynosarge, le gymnase d'Héraclès], en ont tiré une morale et une logique : penser relève moins de l'enchaînement des idées et de la parole que de leur «enchiennement » [Fontenay, 1998: 155] : refus de la tradition, des conventions, etc.). il y faut le mouvement des corps, la métamorphose, la ductilité des formes, une capture et une élaboration des biologies qui font un projet figuratif. Un « zoo-humanisme » (Pierre, 1999 : 31) dont j'ai rappelé comment Souriau, parmi d'autres, l'avait posé au fondement de l'entreprise cinématographique.

On peut trouver dans cette ultime catégorie du «chien jaune» trois registres principaux crescendo :

\section{1. DeMille : des chiens à la tête des hommes}

31 Écartons d'emblée une fausse piste. Certains chiens à tête d'homme ne sont que l'envers $d u$ cynocéphale de tout à l'heure. On peut les voir principalement dans des films de science-fiction ou fantastiques. Dans Mars Attacks! (1995) de Tim Burton, la tête d'une journaliste, Nathalie Lake (Sarah Jessica Parker), est greffée par des extraterrestres potaches sur le corps de son chihuahua. Dans L'Invasion des profanateurs (Invasion of the Body Snatchers, 1978) de Philip Kaufman, Elizabeth (Brooke Adams) et William (Donald Sutherland), alors qu'ils essaient discrètement de s'enfuir de la ville envahie par des extraterrestres cloneurs, croisent dans la rue un chien dont la tête est celle d'un vieillard: sans doute un ratage dans les programmes de réplication. Le maquillage, le collage, l'insert, plus tard le numérique décident généralement de leur sort, qui, pour montrer ce qu'il arrive à des corps dans l'image (et encore plutôt pauvrement : sur la seule modalité de l'addition externe, non de la composition interne - alors que le discours y vise très souvent les périls de la manipulation génétique), ne donnent encore rien à voir de ce qui arrive à l'image.

Un cas un peu plus intéressant et subtil se rencontre chez Cecil B. De Mille. Dans Les Naufrageurs des mers du sud (Reap the Wild Wind, 1942), Steve Tolliver (Ray Milland) est l'un des soupirants de Loxi Clairbone (Paulette Goddard), et l'avocat de la compagnie de transport maritime pour laquelle travaille son rival, Jack Stuart (John Wayne). L'action 
se situe autour de 1840 dans la région des Keys, au large de la Floride. Lors de leur première rencontre à Charleston, au cours d'une réception mondaine, Steve étonne Loxi avec son yorkshire parlant. Bien entendu, le chien ne parle pas : il ne s'agit ni plus ni moins que de ventriloquie. Steve remue avec sa main les mâchoires de l'animal, qu'il porte dans ses bras, pendant qu'il prononce les mots idoines en déformant sa voix. Tolliver et son chien Romulus - le chien porte un nom historique d'être humain, qui en dit long sur les ambitions de son propriétaire (tout au contraire, le nom de la fiancée ne ressemble en rien à un prénom de femme usuel) - forment un couple inséparable. Du moins au début: au fur et à mesure de l'avancée de l'histoire d'amour avec Loxi, le chien disparaîtra du champ, jusqu'à quitter totalement le film sans autre forme de procès, une fois que la place de Loxi comme compagne de Tolliver sera garantie dans le récit. Le chien vaut ici en lieu et place de l'être humain, dont il prépare la place à venir.

Dans Les Conquérants d'un nouveau monde (Unconquered, 1947) du même DeMille, le rôle du chien est le même - mais à l'inverse. Nous sommes dans les années qui précèdent la guerre d'Indépendance. Abigail (Paulette Goddard) et le capitaine Holden (Gary Cooper) ont fui les Senecas qui voulaient mettre la jeune femme à mort. Après avoir leur avoir échappés dans une chute de rapide, ils trouvent refuge dans un cabanon en plein forêt. Les habitants - un couple et leur fille - sont apparemment partis se réfugier au fort le plus proche : seul un petit chien de compagnie est resté sur place. Abigail et Holden s'installent, mais le lendemain matin, le chien conduira Holden à l'endroit où gisent les cadavres de ses maitres. Ils ont manifestement été torturés par des indiens. DeMille ne nous en montrera rien (nous n'aurons droit qu'aux contrechamps, révulsés de Holden, effrayés d'Abigail : bienséance oblige), une poupée trouvée sur place n'en aura que plus de poids visuel, mais surtout le chien sera également, à partir de là, totalement avalé par le hors-champ : seul nous parviennent ses gémissements. Quand les corps humains sont découverts, le chien disparaît avec eux, et si nous ne pouvons pas les voir, alors il nous faut ne plus pouvoir le chien non plus. Ce chien, qu'on avait déjà commencé d'entendre avant de le voir (Abigail l'avait d'abord pris pour un loup...), qu'est-il d'autre sinon le lémure plaintif des êtres humains morts dans d'atroces souffrances et qui ne seront apaisés que lorsque Holden creusera leurs sépultures chrétiennes? Nous ne reverrons le chien, dans cette scène, que pour un plan ultime où on le voit tirer par la gueule la poupée sur le sol : il a désormais pris la place auparavant occupée par fillette vis-à-vis du jouet.

Si, dans Les Naufrageurs des mers du sud, le chien est chassé du film dès que la femme, dont il a dessiné par anticipation les contours dans les bras de l'homme, occupait dorénavant la place de l'animal (physiquement, symboliquement), dans Les Conquérants d'un nouveau monde, il le quitte une fois qu'il est certain que ses maitres ne pourront plus jamais réapparaître dans la moindre image, pour ne revenir très fugacement que comme celui qui, glissant ses crocs dans le vide laissé par les mains d'un enfant, occupe désormais la place de l'être humain. Les deux cas sont très différents, mais la constante est que chez DeMille, du moins dans ces deux films proches dans le temps, la figure du chien - de préférence un chien de compagnie, c'est-à-dire des espèces les plus domestiques - commande à ce qui sera fait visuellement des êtres humains auxquels il est attaché. Une étude précise des autres films de DeMille dans lesquels des chiens jouent un rôle important - Le Signe de la croix (Sign of the Cross, 1932), par exemple, ou encore la première version des Dix Commandements (The Ten Commandments, 1923) serait, à ce titre, passionnante, mais impossible ici. 


\section{2. Tarkovski : à corps de chien}

On trouve de nombreux chiens également chez Andreï Tarkovski, mais des chiens beaucoup plus gros, des chiens que l'on ne peut pas porter dans ses bras: des chiens dont la taille est rapportable à celle de l'homme. Généralement des chiens-loups : celui rencontré par le « stalker » dans la Zone algale, et qui lui restera attaché (Stalker, 1972) ; celui de Kirill qui se détache et court vers un maître cruel qui l'abattra (Andreï Roublev, 1969).

Dans Nostalghia (1983), le poète Andreï Gortchakov (Oleg Yankovski) est un homme solitaire, taciturne, mutique, voûté, exilé russe parti à la recherche en Italie d'un musicien mort et lui-même exilé, qui a perdu le goût de vivre. Tout au long du film, il ne cesse de laisser filer les rencontres : il refuse de voir la Madona del parto de Piero della Francesca, d'aimer son interprète Eugenia, d'entrer en empathie avec la folie de Domenico... Si nous rencontrons au moins une autre espèce de chien (le cocker noir et blanc de la "dame au petit chien »), tout le récit - et l'image, nous verrons - est de nouveau caractérisé par la présence insistante du berger allemand. Celui de la famille restée en Russie, que l'on aperçoit dès le premier plan, avec lequel joue plus tard la fille de Gortchakov au bord d'une mare, etc., et qui est d'abord, comme tous les souvenirs de Gortchakov, filmé en sépia ${ }^{13}$. Mais Nostalghia, qui n'est qu'un film sur un homme qui se souvient, tient absolument tout pour du souvenir en puissance. «Les personnages [de Tarkovski] ainsi ne se souviennent pas; ils se souviennent d'un souvenir » (De Baecque, $1989: 79)$.

37 Prenons la scène dans la chambre d'hôtel. Gortchakov vient de s'allonger sur son lit. Son chien russe sort alors de la salle de bain pour venir s'étendre sur le sol à côté de lui (les chiens tarkovskiens s'approchent souvent d'hommes étendus ou endormis). L'écrivain le caresse dans un état de demi-conscience. Évidemment, il ne s'agit pas de prendre l'arrivée du chien au pied de la lettre : ce qui sort de la salle, c'est un souvenir, un rêve venu par les plis et replis du montage. Le souvenir est une forme de rêverie éveillée qui suppose un certain relâchement de la conscience au monde. L'une des particularités que l'on ne peut manquer de remarque, c'est que le chien est désormais filmé en couleur, c'est-à-dire apparemment pas comme un souvenir (en sépia). Alors quoi ? Mais la couleur ici signale que nous avons moins affaire à une image mentale une sorte de discours indirect libre - qu'au souvenir survenant de lui-même dans l'image, de manière totalement dépsychologisée, moins représenté par l'esprit humain que se présentant dans le monde parmi les autres objets possibles de la sensation : si tout est souvenir, et souvenir de souvenir, alors nous ne percevons jamais que des souvenirs là où nous croyons percevoir des choses. Dès lors, la frontière entre la mémoire et la réalité ne peut plus être que perméable. Notre scène multiplie les associations figuratives sur la logique «marabout-bout d'ficelle» de l'inconscient : le chien entre par la porte de la salle de bains quand il se met à pleuvoir et vient s'allonger sous la fenêtre par laquelle l'eau s'infiltre et forme une flaque sur le sol, marquant visuellement la porosité entre monde extérieur et monde intérieur, à savoir entre veille et endormissement, réalité et mémoire ${ }^{14}$. Le chien - toujours associé chez Tarkovski à des espaces ou des objets aqueux ou humides - fonctionne à l'écran moins comme l'image d'un chien zoologique que comme la signature objectivée du travail de la mémoire (de son travail «liquide» : la mémoire ne va pas d'un point à l'autre en ligne droite, mais s'écoule, déborde, délire étymologiquement : sort du sillon tracé dans la terre - de toutes parts par associations 
et digressions). Le chien est moins une figure photographique qu'une valeur graphique. La chambre d'hôtel dans la pénombre n'est pas qu'une matérialisation de la camera oscura du fors intérieur des images mentales, elle n'est même pas cela du tout: c'est d'abord un emprisonnement figuratif dans l'appareil photographique du cinéma analogique. La composition du plan ne peut que le mettre en abyme : l'incident visuel déterminant est ici cet amas de noir, entre homme et chien, d'homme et de chien, mihumain, mi-animal, ni l'un ni l'autre. Ce noir ne relie pas de l'extérieur deux corps distincts seulement bouturés (comme dans Mars Attacks!, par exemple), mais il spécie chien et homme comme ayant toujours été des événements d'un seul et même corps "protoplasmique " (Tarkovski rejoindrait ici Eisenstein : c'est suffisamment rare pour être souligné15). Cinématographique, ce régime canin des images l'est plus que le précédent en ce qu'il n'est rendu possible que par des astuces d'éclairage de plateau.

\section{3. Ozu : image canine, chien-cyborg, humanité minoritaire}

Richie a beaucoup parlé des fameux «plans-tatami» dans des films comme Fin d'Automne (Akibiyori, 1960) ou Voyage à Tōkyō (Tōkyō monogatari, 1953) - l'expression a fait fortune (Richie, 1980). Ces images, très représentatives du style ozuesque (avec l'aide de Yūharu Atsuta, son opérateur attitré), présentent les caractéristiques principales suivantes : position de la caméra près du sol (presque prostrée), frontalité et statisme du cadrage, axe de l'objectif perpendiculaire au mur du fond, personnages filmés de face (seul ou en plan d'ensemble) et au milieu du cadre, simplification de la composition, dépouillement du champ de vision.

Si l'on a beaucoup insisté sur l'« anti-cinéma» (Yoshida, 2004) d'Ozu - refus de la transparence classique, conscience exacerbée de la présence de la caméra, etc. -, qui cherche moins à monter qu'à laisser voir, on peut également remarquer ceci : Richie rapporte que Sadao Yamanaka, le cinéaste ami d'Ozu précocement disparu, parlait à propos des plans-tatami d'images vues depuis « l'œil d'un chien » (Richie, $1980: 118$ ). La caméra est traitée dans ces plans comme un chien, elle offre le point de vue interne à l'espace domestique, celui de l'animal de compagnie, dont on finit par oublier qu'il est là mais qui a toujours un ceil sur tout, et qui constitue sans doute le point de vue idéal pour des récits de plus en plus réduits à la trame familiale de la vie quotidienne sans péripéties factices ni rebondissements controuvés. Le chien n'est jamais filmé chez Ozu (il y a très peu d'animaux dans ses films : souvent, comme l'oiseau dans le ciel de Un fils unique [Hitori musuko, 1936], on les entend sans les voir...), car c'est lui qui finit par être l'émissaire du regard. Dans ces plans, tout contribue visuellement à produire un effet d'aplatissement de l'image vidant l'espace de sa consistance, de sa profondeur, montrant que l'image n'est qu'une image dans la mesure où l'artifice nous amène à percevoir ce qui se tient derrière les personnages comme s'il se trouvait au-dessus d'eux. Nous voyons depuis la taille d'un petit être vivant, en légère contre-plongée, plus ou moins à ras du sol, d'un regard qui nous rappelle que nous sommes humains par 
l'animalité que nous conservons malgré tout, enfouie sous la langue et la culture, dans notre physiologie perceptive.

N'est-ce pas d'ailleurs ce que très tôt on a salué des puissances inhumaines de la caméra grâce à laquelle l'œil humain « devient tantôt comme un œil fixe à facettes, comme un multiple d'yeux, dont chacun possède une perspective particulière; tantôt comme un œil mobile d'escargot, un œil monté sur une tige extensible et rétractile, un œil pouvant recueillir ses informations, non plus toujours à distance plus ou moins fixe, plus ou moins grande, mais aussi au proximum de la visibilité, presque au contact de l'objet, et pouvant maintenir ce contact si l'objet se déplace » (Epstein, $1974: 146)$ ? Le cinéma augmente notre perception car il nous construit pour la première fois une perception animale (chez l'homme, toute perception est théorique). La caméra-chien d'Ozu n'est pas une figure visible mais une figuration qui rend visible: non seulement le cinéma a ouvert la modernité en faisant de nous des cyborgs (des machines-cyclopes, des centaures-machines: l'histoire de la théorie du cinéma ne manque pas de tels hybrides), c'est-à-dire des « chimères, hybrides de machines et d'organismes théorisés puis fabriqués " (Haraway, $2007: 31$ ), mais nous sommes des hommes parce que nous pouvons percevoir comme des chiens-cyborgs. Pour Haraway, «tant les cyborgs que les espèces de compagnie combinent sous des formes surprenantes humain et non-humain, organique et technologique, carbone et silicium, autonomie et structure, histoire et mythe, riches et pauvres, État et sujet, diversité et déclin, modernité et postmodernité, nature et culture » (Haraway, 2010 : 12). L'animalité compagne ou le cyborg tous deux sont une "coagulation historique du machinique et de l'organique» (idem: 29), le cinéma nous a très tôt incités - avant l'écologie, par exemple, dont il est l'un des plus proches parents - à penser en racontant des histoires notre humanité élargie, c'est-à-dire enfin minoritaire, de part et d'autre du seul cas humain idéologique. Pour en revenir à Ozu : si «peu de cinéastes (...) ont porté (...) la reproduction cinématographique du monde à cette limite : sa réduction à ce que l'on voit, dépouillé de la profondeur et des arrière-mondes historiques, imaginaires, religieux ou métaphysiques " (Ishagpour, 2002 : 15), s'il « réussit à rendre visible que l'homme est un animal parlant (et) hausse au niveau de l'aventure ce qui se passe dans l' infra-ordinaire » (Sobel et Gresh, 1993: 123), c'est parce que rares sont les cinéastes à être parvenus à une telle image canine, c'est-à-dire à une perception anthropocéphale exigeant de l'homme qu'il regarde désormais les images, non pas comme un chien voit, mais comme un homme voit, un homme qui ne peut plus être homme que parce qu'il est un animal qui s'est redressé. C'est-à-dire accroupi dans le chien qui ne cesse de contribuer à le façonner, à en inventer tous les régimes de fiction.

\section{BIBLIOGRAPHIE}

ALIZART, Mark (2018). Chiens. Paris : PUF, coll. « Perspectives critiques ».

BELLOUR, Raymond (2009). Le Corps du cinéma. Hypnoses, émotions, animalités. Paris : P.O.L, coll. «Trafic ». 
BUNYAN, John (1842). A Few Sighs from Hell, or « The Groans of the Damned Soul » [1658], The Practical Works of John Bunyan, vol. v. Londres : Hamilton, Adams \& Co..

BURKE, Edmund (1990). Recherche philosophique sur nos idées du sublime et du beau, trad. fr. Baldine Saint-Girons. Paris : Vrin, coll. « Bibliothèque des textes philosophiques ».

CAVELL, Stanley (1993). À la recherche du bonheur. Hollywood et la comédie du remariage, trad. fr. Christian Fournier et Sandra Laugier. Paris : Cahiers du cinéma, coll. « Essais ». соцомв, Christophe (1984). La Découverte de l'Amérique, I. Journal de bord. 1492-1493, trad. fr. Michel Lequenne. Paris : Maspero/La Découverte.

DE BAECQUE, Antoine (1989). Tarkovski. Paris : Éditions de l'Étoile/Cahiers du cinéma, coll. « Auteurs".

DeLEUZE, Gilles et GUATTARI, Félix (1991). Qu'est-ce que la philosophie ?. Paris : Éditions de Minuit, coll. «Critique».

Deleuze, Gilles et GUATTARI, Félix (1980). Mille Plateaux. Paris : Éditions de Minuit, coll. « Critique ». DELEUZE, Gilles et PARNET Claire (1977). Dialogues. Paris : Flammarion, coll. « Dialogues ».

DURAFOUR, Jean-Michel (2013). Brian De Palma. Épanchements : sang, perception, théorie. Paris :

L'Harmattan, coll. « Esthétiques ».

DURAfour, Jean-Michel (2018). Cinéma et cristaux. Traité d'éconologie. Paris : Mimésis, coll. «Images, médiums ».

EISENSTEIN, S. M. (2013). Walt Disney, trad. fr. André Cabaret. Paris : Circé.

EPSTEIN, Jean (1974). « Le monde fluide de l'écran » [1950], Écrits sur le cinéma (1921-1953). Paris :

Seghers, coll. « Cinémaclub».

FONTENAY, Elisabeth de (1998). Le Silence des bêtes. La philosophie à l'épreuve de l'animalité. Paris :

Fayard.

HARAWAY, Donna J. (2010). Manifeste des espèces de compagnie. Chiens, humains et autres partenaires, trad. fr. Jérôme Hansen. Paris : Éditions de l'Éclat, coll. « Terra incognita ».

HARAWAY, Donna J. (2007). Manifeste cyborg et autres essais. Sciences - Fictions - Féminismes, éd. par Laurence Allard, Delphine Gardey et Nathalie Magnan. Paris : Exils, coll. « Essais ».

ISHAGHPOUR, Youssef (2002). Formes de l'impermanence. Le style de Yasujirō Ozu. Paris : Farrago/Lé Scheer.

PIERRE, Sylvie (1999). « L'Indien, le chien et le cheval de fer », Vertigo, dossier « Animal », n 19.

PLATON (1993). Le Sophiste, trad. fr. Nestor Cordero. Paris : Flammarion, coll. « GF ».

QUIGNARD, Pascal (2009). La Barque silencieuse. Paris : Éditions du Seuil.

RICHIE, Donald (1980). Ozu, trad. fr. Pierre Maillard. Genève : Lettre du blanc.

SIMENON, Georges (1931). Le Chien jaune. Paris : Librairie Arthème Fayard.

SOBEL, Bernard et GRESCH, Sylviane (1993). Un art légitime. Arles : Actes Sud, coll. « Le temps du théâtre ».

SOURIAU, Étienne (1956). «L'univers filmique et l'art animalier », Revue internationale de filmologie, vol. 7-8, n² 25, janvier-mars 1956. 
THOMAS, Keith (1985). Dans le jardin de la nature. La mutation des sensibilités en Angleterre à l'époque moderne (1500-1800) [1983], trad. fr. Catherine Malamoud. Paris : Gallimard, coll. « Bibliothèque des Histoires ».

THOMPSON, Charis (2005). Making Parents. The Ontological Choregraphy of Reproductive Technologies. Cambridge (États-Unis)/Londres : The MIT Press, coll. « Inside Technology ».

YoSHIDA, Kijū (2004). Ozu ou l'anti-cinéma, trad. fr. Jean André Viala. Arles : Actes Sud, coll. «Institut Lumière ».

\section{NOTES}

1. Expression deleuzienne (Deleuze et Parnet, $1977: 8$ ).

2. Il y va aussi, dans cette relation à la chienne, d'un certain rapport aux « terres expropriées des Amérindiens » (idem : 10).

3. Pour plus de détails sur ce vocabulaire et sur la pensée des images qui le sous-tend, voir Durafour, 2018.

4. J'emprunte cette formule à Quignard, 2009 : 104.

5. Et « chien » peut ainsi devenir la pire des insultes : «De tous les animaux, les chiens sont en vérité les plus sociaux, les plus affectueux et les plus aimables ; mais l'amour approche beaucoup plus du mépris qu'on ne l'imagine communément; ainsi nous avons beau caresser les chiens, nous leur empruntons une appellation des plus injurieuses, quand nous voulons marquer notre opprobre; et cette dénomination exprime dans toutes les langues la dernière abjection et le dernier mépris » (Burke, 1990 : 109-110).

6. L'autre ligne de l'intrigue met en scène un léopard brésilien tout autant symbolique - le « bébé » du titre - dans la mesure où l'espèce est exclusivement africaine ou asiatique.

7. Je reprends ici très succinctement la lecture proposée par Cavell, 1993. - Chez Hawks, l'animal, vu ou seulement dit, sert souvent d'intermédiaire pour signaler une correspondance sexuelle (la course hippique dans Le Grand Sommeil [The Big Sleep, 1946], par exemple).

8. Manger des chiens a longtemps été une pratique culinaire appréciée en Corée (le bosintang), depuis l'époque des Trois Royaumes, avant de devenir un motif de conflit éthique entre la Corée du Sud et le reste du monde (Anthony L. Podberscek, 2009 : 619). Le sujet est notamment revenu sur le devant de la scène lorsque la Corée du Sud a été choisie pour accueillir la Coupe du monde de football en 2002. Adresse inconnue a été réalisé dans ce climat médiatique. Le film montre, sans contrition mais aussi sans vouloir condamner (c'est l'une de ses faiblesses), différents comportements à l'égard des chiens: l'élevage alimentaire brutal, la cohabitation avec les animaux errants, l'animal de compagnie, l'identification (Chang-guk bat sa mère mais est incapable de battre un chien)...

9. La victima latine est d'abord une bête, certes, mais en tant qu'elle est offerte en sacrifice aux dieux, c'est-à-dire recouverte par le droit.

10. J'y reviens autrement plus loin : la seule humanité compatible avec le respect des autres formes de vie, la seule humanité viable est une humanité pensée comme minorité.

11. Mon recours à Deleuze se limitera ici à ces propositions. Le devenir-animal chez Deleuze et Guattari privilégie les animaux de "meute», de «contagion» (Deleuze et Guattari, 1980 : 296-297), au détriment des animaux «œdipiens» (Deleuze et Guattari, 1980: 294) et individualisés comme les chiens. Deleuze n'a jamais caché son aversion pour les animaux de compagnie.

12. Le sophiste ressemble au philosophe « comme le loup au chien ».

13. Il y aura ensuite un autre berger allemand : celui de Domenico, sur lequel je ne dirai rien. Il vient, évidemment, provoquer des régimes de superposition et de confusion avec le chien 
familial qui complexifient le travail de l'image, mais se rapprochent aussi plus de la manière, sédimentaire, concentrique et anachronique, dont fonctionne notre mémoire.

14. Le plan final, après la mort de Gortchakov, qui le montre avec son chien devant sa datcha familiale à l'intérieur de la cathédrale italienne, en donnera le perspectivisme le plus synthétique en fusionnant passé et présent, au-delà de la vie, dans la monochromie de la mémoire. Une fois mort, toute la vie ne peut plus être qu'un souvenir.

15. Pour Eisenstein, les formes dérivent d'un "protoplasme originel qui n'avait pas encore de forme "stabilisée" mais état apte à en prendre une, n'importe laquelle, et, d'échelon en échelon, à évoluer jusqu'à se fixer dans n'importe quelles - dans toutes les - formes d'existence animale » (Eisenstein, 2013 : 27).

\section{RÉSUMÉS}

Cet article se propose d'étudier, dans l'histoire du cinéma et à travers des exemples choisis (De Sica, Hawks, DeMille, Kim Ki-Duk, Tarkovski, Ozu), les manières dont les puissances filmiques peuvent s'emparer de la représentation conjointe de l'homme et du chien. Trois catégories vont se distinguer tout particulièrement : 1/ le chien " anthropologique " associé à des vertus ou des vices humains et symbolisant un état moral distinctif de l'homme; 2/ le chien " anthropomorphologique» comme symptôme du corps humain en tant que corps animal et pathique; 3/ le chien "anthropocéphale ", l'homme comme un chien à tête d'homme, qui culmine dans la qualification canine de l'appareil de prise de vues. Ces catégories, évidemment perméables les unes les autres dans les faits, entendent dessiner une première voie d'accès esthétique à la figuration symbiotique de l'espèce humaine et de l'animal.

This article is dedicated to the study, in the history of cinema and through selected examples (De Sica, Hawks, DeMille, Kim Ki-Duk, Tarkovsky, Ozu), of the ways in which the filmic mightness can seize the joint representation of man and dog. Three categories will stand out in particular: 1 / the "anthropological" dog associated with human virtues or vices and symbolizing a distinctive moral state of mankind; 2 / the "anthropomorphological" dog qua a symptom of the human body as an animal and pathic body; 3 / the "anthropocephalic" dog, the man like a dog with a man's head, which culminates in the canine qualification of the camera. These categories, obviously permeable to each other, intend to draw a first aesthetic access to the symbiotic representation of the human species and the animal.

\section{INDEX}

Mots-clés : cinéma, figuration, esthétique, « image canine », " anthropocéphalie »

Keywords : cinema, figurative art, aesthetics, « dog image », anthropocephalia » 
AUTEUR

JEAN-MICHEL DURAFOUR

Aix-Marseille Université

jean-michel.durafour[at]univ-amu.fr 